\title{
Dentin Biomodifiers to Stabilize the Bonded Interface
}

ISSN: 2637-7764

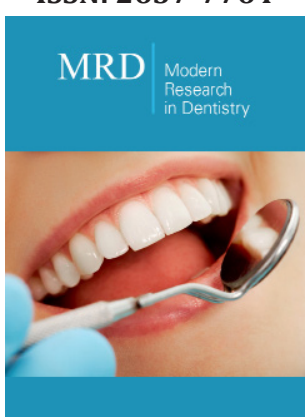

*Corresponding author: Rajni Nagpal, Department of Conservative Dentistry and Endodontics,Kothiwal Dental College and Research Centre, Moradabad, UP 244001, India

\section{Submission: 此 March 06, 2020}

Published: 陋 November 24, 2020

Volume 5 - Issue 5

How to cite this article: Rajni Nagpal, Meenal Agarwal, Nida Mehmood, Udai Pratap Singh. Dentin Biomodifiers to Stabilize the Bonded Interface. Mod Res Dent. 5(5). MRD. 000622. 2020.

DOI: 10.31031/MRD.2020.05.000622

Copyright@ Rajni Nagpal, This article is distributed under the terms of the Creative Commons Attribution 4.0 International License, which permits unrestricted use and redistribution provided that the original author and source are credited.

\section{Rajni Nagpal ${ }^{1 *}$, Meenal Agarwal' ${ }^{2}$, Nida Mehmood ${ }^{2}$ and Udai Pratap Singh ${ }^{3}$}

${ }^{1}$ Professor, Department of Conservative Dentistry and Endodontics, Kothiwal Dental College and Research Centre, Moradabad, 244001, UP, India

${ }^{2} \mathrm{PG}$ Student, Department of Conservative Dentistry and Endodontics, Kothiwal Dental College and Research Centre, Moradabad, 244001, UP, India

${ }^{3}$ Professor and Head, Department of Conservative Dentistry and Endodontics, Kothiwal Dental College and Research Centre, Moradabad, 244001, UP, India

\begin{abstract}
Majority of esthetic restorations are currently done using resin composites. It is important to have a stable adhesion between tooth substrate and adhesive resin in order to have clinically successful composite restorations over long term. Even though adhesive systems have significantly improved, still the bonded interface remains the weakest area of resinous restorations. This has been attributed mainly to degradation of exposed collagen at the base of hybrid layer leading to deterioration of resin-dentin bond. Various dentin biomodifiers are currently under research to reduce the enzymatic breakdown of collagen and improve their mechanical stability. Dentin biomodification involves use of matrix metalloproteinase inhibitors and collagen cross linkers on acid conditioned dentin. Although only preliminary research is available, dentin biomodification appears to be a promising approach to stabilize the resin-dentin bond. This paper briefly reviews different dentin biomodifying agents, their mechanism of action and their role in improving the resin-dentin durability.
\end{abstract}

Keywords: Resin-dentin bond; Matrix metalloproteinase inhibitors;Collagen cross linkers;Bond durability

\section{Introduction}

Dental caries is prevalent all around the world, and resin composites are widely used as filling materials [1]. However, longevity of resinous restorations is not satisfactory with failure rate ranging from $15 \%$ to $50 \%$ [2]. In addition, resin-dentin bonds are less durable than resin-enamel bonds, because of the heterogeneity of the structure and composition of dentin [3]. The failure of resin-dentin bonding results in microleakage, staining, recurrent caries, and postoperative sensitivity [4], and the interaction of these situations can further accelerate the degradation of the bond. Even though adhesive systems have significantly improved, the bonded interface remains the weakest area of resinous restorations [5]. Improving the chemical and mechanical stability of the collagen fibrils within the hybrid layer may be of clinical importance to enhance resin-dentin bond durability. This may be achieved by stabilization of dentin collagen with biocompatible cross-linking agents to increase mechanical properties and decrease enzymatic degradation with matrix metalloproteinase inhibitors (MMPI). Therefore, the aim of this paper is to discuss factors related to failure of resin-dentin bond along with various dentin biomodifying agents currently under research to optimize the longevity of resin dentin bond.

\section{Factors leading to resin- dentin bond failure}

A. Discrepancy between adhesive resin infiltration and dentin demineralization depth: With the etch and rinse systems, the difference between penetration of the adhesive and action of the acid etchant leads to an incomplete hybridization of the exposed collagen network. Therefore, at the base of hybrid layer, collagen fibrils remain uninfused, being more susceptible to hydrolytic degradation. The inability of resin monomers to replace both free and collagen-bound water present in the inter and intrafibrillar compartments does not allow 
to achieve a complete and stable hybrid layer. Additionally, highly hydrated proteoglycan hydrogels found in interfibrillar spaces, act as filters that trap the monomers of large molecules, such as BisGMA, and only allow the passage of small monomers, such as HEMA, toward the base of the hybrid layer. HEMA produces weak linear chains that, when subjected to stresses, lead to failure due to cyclic fatigue of the collagen chains [6].

B. Degradation of exposed collagen: Demineralized dentin collagen matrix acts as a scaffold for resin infiltration during the resin-dentin bonding procedure, forming the hybrid layer [7]. Degradation of collagen matrices by matrix metalloproteinases (MMPs) and cysteine cathepsins is believed to be among the major reasons for the failure of resin restorations [8]. MMPs and cysteine cathepsins can attack type I collagen, the most abundant organic component of dentin. They can be activated by proteinases, chemical agents, low $\mathrm{pH}$, heat treatment, as well as mechanical stress [8]. Acid-etchants used in dentin bonding can uncover and activate matrix-bound MMPs. Incomplete resin infiltration also contributes to their activation. The exposed dentin collagen loses its protective triple helical conformation and presents the recognizable and available cleavage sites, becoming more vulnerable to MMPs and cathepsins [9].

c. Degradation of the Adhesive Resin: The use of hydrophilic monomers in adhesive systems, such as 2-hydroxyethylmethacrylate (HEMA), seeks to improve infiltration of the exposed collagen network, which is inherently humid. This results in an immediate improvement in bond strength [10], but the longevity of this dentin-resin bond is compromised. A weak hybrid layer is generated at the adhesive interface in the presence of water, in which the phenomena of hydrolysis and leaching of resin adhesives occur. Current adhesives include hydrophilic and hydrophobic components that, in aqueous solution, produce nano phase separation of adhesives. The hydrophilic elements penetrate the interior of the hybrid layer, while the hydrophobic monomers remain on the surface thereby leading to inadequate polymerization in the deepest zone of the hybrid layer. The incomplete polymerization of the hydrophilic portion of the adhesives and the aqueous sorption of the material allow the mobilization of water, which forms large aqueous channels within the hybrid layer leading to water tree phenomenon [11].

\section{Dentin biomodification}

Biomodification of dentin substrate involves pretreating it with MMPIs and collagen cross-linkers (CCL) as dentin biomodifiers, to achieve a more stabilized and durable adhesive interface. Dentin biomodification through collagen cross-linking and MMP inhibition can be achieved by either synthetic (carbodiimide, glutaraldehyde, CHX, tetracyclines, quaternary ammonium compounds, ascorbic acid) or natural products (quercetin, baicalein, catechins, oligomeric proanthocyanidins, genipin, hesperidin, and other polyphenols) and products activated by physical methods such as ultraviolet-A activated riboflavin [12].

\section{Matrix metalloproteinase inhibition and collagen cross- linking}

One of the mechanisms of MMP inhibition is cationic-anionic reaction. Negatively charges catalytic sites of MMPs may bind electrostatically with cationic agent like Chlorhexidine (CHX) and block the active site. Chelating or coordinate covalent bond with zinc or calcium present in the catalytic domain also leads to loss of catalytic activities of MMPs. Another mechanism of MMPs inhibition is protein cross-linking, cross-linkers can induce conformational changes in MMP's, 3D structure and hindering molecular mobility which is essential for their enzymatic activity. In addition, activity of MMPs can be interfered by altering circumstances around MMPs like $\mathrm{pH}$ and concentration of $\mathrm{Zn}^{2+}$ [13]. The resin-dentin bond can thus be strengthened by protein cross-linking agents which stabilize collagen matrix and improve the mechanical properties of the hybrid layer.

\section{Dentin biomodifiers}

Glutaraldehyde: Glutaraldehyde (GA) is predominately used as a fixative that cross-links collagenous biomaterials. GA contains two aldehyde groups which are able to react with the amino groups of lysyl or hydrolysyl polypeptide residues in the collagen, thus forming reducible Schiff base crosslinks. It is well accepted that GA can effectively improve the resistance of uncross-linked or mildly cross-linked collagen matrices to degradation by collagenases [14]. By improving dentin's mechanical properties it is expected that glutaraldehyde would also contribute in improving the resistance to bond degradation. However, despite its efficacy glutaraldehyde has been found to be approximately 120 times more toxic than proanthocyanidins [15].

Proanthocyanidins: Proanthocyanidins (PAs) are a class of bioflavonoids that are naturally occurring plant metabolites available in fruits, vegetables, nuts, seeds, flowers and barks. The building blocks of PAs are flavan-3-ololigomers which have 3 rings (ring A: triketide, ring B: phenyl propanoid, ring C: pyran which is formed by condensation). The main flavan-3-ol units in grape seed extract PAs are catechin (C), epicatechin (EC), catechingallate (CG) and epicatechingallate (ECG). PAs have gained popularity in the fields of nutrition ,health, and medicine due to their physiological activities such as antioxidant, antimicrobial, and anti-inflammatory properties, effects on cardiovascular diseases, antiallergic and enzyme inhibitory activities against phospholipase $\mathrm{A}_{2}$, cyclooxygenase, and lipooxygenase [16].

PA has been used in the adhesive and restorative dentistry as a natural collagen cross-linking agent. Several studies have shown that PA functions as dentin collagen matrix stabilizer and improves its mechanical properties and increasing its resistance to biodegradation $[17,18]$. Biomodification of demineralized dentin matrix with PA inhibits the proteolytic activity and stabilizes the adhesive/dentin interface against enzymatic degradation. Thus, incorporation of PA into simplified hydrophilic adhesive systems may be a means to improve the durability of adhesive restorations. 
Chlorhexidine (CHX): CHX, a biguanide antimicrobial agent, has been broadly used in dentistry for its microbial efficacy and substantivity [19]. CHX is the most widely accepted non-specific MMP inhibitor even at a concentration of $0.05 \mathrm{wt} \%$. The mechanism of chlorhexidine on MMP inhibition is probably based upon the cationic-anionic reaction of $\mathrm{CHX}$ on glutamic acid residue of cysteine domain which may deform MMPs molecules and prevent them from binding to substrates [20]. CHX could also bind with calcium and zinc ions to MMPs, resulting in loss of MMP's catalytic activity. CHX not only can inhibit MMPs but also electrostatically binds to demineralized dentin. This may be the reason for the long-term MMP inhibition efficacy of CHX in resin dentin bonds. In addition to adding CHX into primer and bond, $2 \mathrm{wt} \%$ CHX has been incorporated into conventional phosphoric acid. It has been demonstrated that CHX influences the mechanical properties of bonding resin depending on its concentration. CHX digluconate is only available as an aqueous solution, therefore mixing this solution with adhesives may increase water entrapment in the smear layer [21]. For CHX diacetate, it would increase water sorption of copolymers.

EDTA: EDTA acts as a chelating agent, reacting with calcium ions from dentin hydroxyapatite and forms soluble calcium salts. As EDTA is an effective $\mathrm{Zn}^{2+}$ and $\mathrm{Ca}^{2+}$ chelator, it might inhibit MMP activity [22]. 17wt\% EDTA pretreatment of dentin beams for as little as 1 minute significantly lowered the endogenous MMP activity of completely demineralized dentin beams by chelating both the calcium and zinc ions from the enzyme that are necessary for their optimum function.

Galardin: Galardin is a potent and broad-spectrum hydroxamatetype synthetic MMP inhibitor designed as a molecular mimic of MMP substrates, which allows it to enter the active site of MMPs, where it binds the critical zinc atom. Galardin is active against several MMPs. Galardin has potent inhibitory activity against MMP$1,2,3,8$ and 9. Galardin has been shown to reduce the loss of bond strength comparable to the effect of CHX [23].

Chemically modified tetracyclines (Doxycycline and Minocycline): They are considered as broad spectrum MMP inhibitors. Inhibition of MMPs theoretically can occur at numerous levels attributable to the multiple steps involved in MMP transcription, protein synthesis and enzyme activation through binding in the MMP active site. Their inhibitory effects were attributed to their ability to chelate $\mathrm{Ca}^{+2}$ and $\mathrm{Zn}^{+2}$ [24] which are two essential ions for MMPs to maintain their structure and functional active sites. Therefore, by binding to the active site, modified tetracyclines can alter the conformation of the proenzyme (MMP) molecule, thus blocking its catalytic activity in the extracellular matrix. Despite some difference in chemical structure, both minocycline and doxycycline can inhibit collagenases and gelatinases.

Carbodiimide: 1-ethyl-3-(3-dimethylaminopropyl) carbodiimide hydrochloride or EDC is a synthetic cross-linking agent which when applied directly to demineralized dentin has been found to improve the bond strength and structural integrity of the resin/dentin interface over time by preventing the enzymatic and hydrolytic degradation, through the formation of inter and intra-molecular crosslinks [25]. EDC is known as a zero-length cross-linking agent, due to its ability to cross-link peptides to one another without introducing additional linking groups. EDC causes cross-linking to occur in dentin collagen as well as in dentin matrix-bound MMPs. It is found that when collagen cross-linkers are applied on demineralized dentin, cross-linking occurs more rapidly in MMPs as compared to collagen [26]. This could be due to better accessibility of carboxyl and amino groups in MMPs than in collagen. Thus, it becomes evident that EDC is a potent MMPI and its MMP inhibition effect is quicker than its cross-linking effect. The biomodification of collagen by extrinsic cross-linkers like EDC can induce the formation of additional inter and intra-molecular cross-links, increasing the ultimate tensile strength (UTS) and elastic modulus of demineralized dentin.

Chitosan: Chitosan is a cationic polysaccharide produced through the deacetylation of chitin, a polysaccharide found in the exoskeleton of crustaceans, through an alkalinization process under high temperatures. Chitosan showed a significant increase in the resistance to degradation of collagenase after dentin collagen is coated with nanoparticles of chitosan [27]. It also showed increase in the microhardness of the root dentin layer. Chitosan modified with methacrylate as a component of an etch-and-rinse adhesive, is proposed as a system to improve the durability of dental restorations.

Hesperidin: Hesperidin (HPN) is a glycoside flavonoid extracted from citrus fruits, which belongs to the flavanone subgroup. The pharmacological properties and medicinal uses of HPN are associated with its wide range of benefits such as anti-inflammatory, analgesic, anti-microbial, and antioxidant effects. HPN is also capable of carcinogenesis inhibition, bone loss prevention and inhibition of MMPs proteolytic activities. Studies showed the potential of HPN to prevent collagen degradation against proteolytic enzymes and incorporation of $2 \%$ HPN in a self-etch primer significantly improved the immediate and long-term (1-year storage in artificial saliva) micro tensile bond strength [28].

Genipin: Genipin (GNP) is a hydrolytic product of geniposide, which is extracted from the fruit of Gardenia jasminoidesellis. GNP can react with primary amine groups within collagen molecules, thus forming crosslinks. GNP has been tested on bovine dentin and shown to significantly increase the resistance of collagen fibrils against enzymatic digestion in a concentration and time dependent manner. Also, chemical modification of human dentin with GNP resulted in a significant increase in the ultimate tensile strength and increased bond strength in adhesive restorations [29]. However, its pigmentation impacts may lead to the staining of treated dentin surface, which presents a clinical limitation due to aesthetic concerns.

Benzalkonium chloride: Benzalkonium chloride (BAC), a mixture of alkylbenzyl-dimethyl ammonium chlorides of various lengths 
of alkyl chains, is a nitrogenous cationic surface-acting agent containing a quaternary ammonium group. Similarly, to biguanides, BACs are only bound to dentin collagen electrostatically and thus, they may leach from the hybrid layer, consequently losing their autoproteolytic benefits. BAC concentrations of $0.5-1.0 \mathrm{wt} \%$ or more inhibit rhMMP-2, 8 and 9 up to $100 \%$ [30]. Similar BAC concentrations inhibit matrix bound MMPs between 55\% and 76\% for a 30 days period [31]. Thus, BAC can be added to the list of quaternary ammonium compounds that inhibit MMPs in addition to their antibacterial effects.

Riboflavin: Low-dose riboflavin has been tested as dentin crosslinking agent in conjunction with UVA or dental blue light with good success [32]. Riboflavin is apt for crosslinking dentin collagen due to its biocompatibility and its ability to produce free radicals when photo-activated with spectral range from UV to visible light. These free radicals or so called reactive oxygen species such as $\mathrm{O}^{2}$ and $\mathrm{O}^{2-}$ are released when riboflavin is photoactivated and light is absorbed, forming covalent crosslinks between adjacent collagen molecules. Cova et al. concluded that UVA activated riboflavin increased the immediate bond strength to dentin, stabilized the adhesive interface, and inhibited dentin matrix metalloproteinases, thereby increasing durability of resin/dentin bonds [32].

Alpha tocopherol: Vitamin E is generally considered the most important and effective lipid soluble antioxidant. Alpha tocopherol allowed free-radical polymerization of the adhesive resin to proceed without premature termination by restoring the altered redox potential of the oxidized bonding substrate and improve resin bonding [33].

L-ascorbic acid: L-ascorbic acid (AA) is the white to light-yellow, water-soluble vitamin commonly known as vitamin C. Sodium ascorbate (SA) is a salt of AA. Both AA and SA have antioxidant properties. It is known that $\mathrm{AA}$ enhances the dentin bonding strength of adhesive resins when used on dentin surfaces as an experimental conditioner [34]. SA is known to enhance the bonding strength of adhesive resins on dentin surfaces treated with sodium hypochlorite in endodontic and operative dentistry [35].

Quaternary ammonium compounds (QAMs): Quaternary ammonium compound (QAM) is a non-volatile and chemically stable polymer that is not permeable through the oral mucosa in contrast to the main problems of the chemically related quaternized monomers. Polymerizable quaternary ammonium methacrylate's (QAMs), especially 12- methacryloyloxy dodecyl 1- pyridinium bromide (MDPB) have been incorporated into selfetching primers because they possess antimicrobial properties and can copolymerize with adhesive monomers. QAMs including MDPB demonstrate inhibitory effects on both soluble rhMMP-9 and matrix-bound MMPs [36]. The cationic quaternary ammonium methacrylate's (QAMs) may electrostatically bind to negatively charged catalytic sites of MMPs, which contain cysteine-rich repeats, blocking the active site, in addition to its ability to kill bacteria.
Bromelain enzyme: Bromelain is a proteolytic enzyme (proteases) which belongs to a group of protein digesting enzymes obtained commercially from the fruit or stem of pineapple. Hydrolysis of proteins to yield amino acids is catalyzed by the proteases. Bromelain enzyme can reduce nano leakage after collagen removal. This could be because of the depletion of collagen from the surface of acid-etched dentin resulting in increased permeability of dentin substrate due to the enlargement of dentinal tubules near the outer dentin surface. This facilitates the spread and diffusion of adhesive monomers through dentin. The surface energy of the dentin is improved, because the hydroxyapatite has a high surface energy substrate while collagen has a low energy surface, and this leads to enhanced diffusion of adhesive monomers through dentin [37].

EGCG (Epigallocatechin-3-0-(3-0-methyl) gallate): EGCG is a potent inhibitor of MMPs and cysteine cathepsins, and their inhibitory effect is concentration-dependent. First, EGCG binds to zinc ion, which plays an important role in protection collagen against degradation by creating a new conformation, protecting the cleavage sites of collagen from metalloproteinases. Second, it causes irreversible degradation of the MMP-2 molecule by exhibiting hydrogen bonding and hydrophobic interactions with it, or by masking the catalytic region of MMP-2 [38]. More than $50 \%$ of green tea contains EGCG, the main polyphenol component. To improve the stability and bioavailability of EGCG and endowed EGCG with new biological functions, researchers have tried to introduce new function groups to EGCG by chemical synthesis, such as acetylated EGCG, methylated EGCG, and glycosylated EGCG. When EGCG and EGCG-3Me were each incorporated into the adhesive, the resin-dentin interface was protected from thermocycling effects resulting in improved bonding. EGCG and EGCG-3Me modified adhesives showed effective antibacterial activity against $S$. mutans. adhesive modification by EGCG-3Me at a concentration of $400 \mu \mathrm{g} /$ $\mathrm{mL}$ may be a more promising method to improve the long-term use of resinous restorations [38].

Curcumin: Curcumin is a well-known, non-toxic polyphenolic compound extracted from the rhizome of the plant Curcuma longa. Curcumin, isolate from the natural extracts, contains three major curcuminoids namely curcumin, demethoxycurcumin, and bisdemethoxycurcumin. The interaction of curcumin with MMPs can be explained by their ability to chelate the catalytic $\mathrm{Zn}^{+2}$ ions essential for MMP activity via $\beta$-diketone zinc-binding site of curcumin similar to the tetracycline based MMP inhibitors. The $\beta$-diketone form contains an activated carbon in the heptadienone linkage between the two phenolic rings, due to the delocalisation of the unpaired electron on the adjacent oxygens of this carbon. At acidic and neutral pHs (between $\mathrm{pH}$ : 3-7), the $\mathrm{C}-\mathrm{H}$ bonds are weak on this carbon, and this allows curcumin to serve as a potent $\mathrm{H}$ donor. Additionally, metal cations can be released from proteins [39]. In mildly acidic conditions, curcuminoids contributes to this process by chelating and removing metal ions from metalloproteins treatment of collagen with curcumin increases aggregation of collagen depending on curcumin concentrations. The treatment of dentin collagen by curcuminoids can make them more resistant 
to endogenous protease-mediated degradation by crosslinking the collagen fibrils. The interaction of curcumin with collagen consists of hydrogen bonding and electrostatic charge interactions.

Rosmarinic acid: Rosmarinic acid (a-o-caffeoyl-3,4dihydroxyphenyllactic acid) is a diphenolic compound that contains two catechol (1,2-dyhydroxy- benzene) rings, contributing to rosmarinic acid's polarity [40]. Rosmarinic acid has an MMPinhibitor effect in addition to its antioxidant activity. Inhibition of MMP-2 and -9 activity may slow caries progression thereby increasing the durability of resin-dentin bonds [40]. The crosslinking effect of rosmarinic acid is attributed to its interaction with proline-rich proteins, such as collagen. A natural cross linker may improve dentin bonding, because the cross-linked collagen matrix exhibits increased mechanical properties and resistance to proteolytic degradation. Moreover, the advantage of rosmarinic acid is the presence of four phenolic hydrogens $(-\mathrm{OH})$ which contribute to controlling free radical oxidation. The -OH group in phenol acts as a chain-breaking antioxidant because it scavenges reactive radicals. The resulting radicals tend to be poorly reactive because of electron delocalization into the aromatic ring, so that the reactive radical is replaced by one of limited reactivity.

Cashew Nutshell Liquid: Cardol and cardanol correspond to more than $95 \%$ of the composition of technical cashew nutshell liquid produced in the industries. The presence of a long 15-carbon alkyl side chain provides additional hydrophobic interaction with dentin collagen fibrils which may contribute to dentin biomodification. Cardol and cardanol are non-cytotoxic compounds in low concentrations possessing antioxidant effects, they present inhibition of matrix metalloproteinase- 2 and matrix metalloproteinase-9 [41]. The lower molecular weight of cashew nutshell liquid causes it to attain greater and faster penetration in dentin collagen matrix, thus explaining the highest increase in the bond strength. Moreira et al reported that CNSL yield best biomodification outcomes when applied for one minute without staining the dentin collagen [42].

Quercetin: Quercetin belongs to the flavanol group and is commonly found in onions, apples, tea and red wine. As a result of its cross-linking properties, QC has been shown to enhance the mechanical properties and thermal denaturation temperature of the extracellular matrix of heart valves. Quercetin offers several benefits, including anti-inflammatory, antioxidant and cancer preventing properties. Quercetin could inhibit the activity of MMP-2 and MMP-9 in PC-3 cells [43]. Quercetin can cross-link with collagen to decrease the formation of water canals by resisting collagenase attack and strengthen its stability. Being a natural cross-linker, quercetin has been widely accepted for its biocompatibility and safety.

Baicalein: Baicalein (BAI) is one of the major flavonoids in Scutellaria baicalensis. Because of its potential MMP inhibition and cross-linking abilities, BAI is capable of stabilizing collagen fibrils and protecting the integrity of the hybrid layer in dentin bonding.
Since the hydroxyl groups of BAI can form hydrogen bonds with the amide carbonyl of proteins, it can act as a potential cross-linker. Furthermore, it has been reported that cross-linkers can cross-link not only proteins but also proteases. This may directly interfere with the molecular mobility of proteases or inactivate C-terminal telopeptidases, maintaining telopeptides ability and sterically blocking collagenase binding to the critical peptide bond [44]. Therefore, the MMP inhibitory mechanism of BAI in endogenous dentin-bond MMPs might be as follows. First, BAI could compete with the active center of the enzyme through a metal-chelating effect, grabbing metal irons such as $\mathrm{Zn}^{2+}$ to suppress the activity of MMPs. Second, BAI might cross-link and alter the three-dimensional structure or molecular mobility of MMPs, resulting in the loss of the collagen enzymolysis ability of the latter. Third, BAI might crosslink with dentin collagen fibers through hydrogen bonds, changing or covering the recognition sites of MMPs in collagen to interfere with enzymatic coordination and complexation, thereby protecting noncoated collagen from degradation.

\section{Conclusion}

Success in adhesive dentistry means long lasting restorations. As composite restorations are the most popular materials to deal with caries nowadays, it is very important to understand the issues related to resin-dentin bond durability. Although various studies have investigated the role of MMPI and collagen cross-linkers in enhancing the longevity of resin-dentin bonding, still more research is required to develop new bonding systems that provide durable MMP-inhibitory and collagen crosslinking functionality to stabilize the hybrid layer and thereby improve durability of adhesive composite restorations.

\section{References}

1. Zhou W, Liu S, Zhou X, Hannig M, Rupf S, et al. (2019) Modifying adhesive materials to improve the longevity of resinous restorations. Int J Mol Sci 20(3): 723.

2. Opdam NJ, Bronkhors EM, Loomans BA, Huysmans MC (2010) 12-year survival of composite vs. amalgam restorations. J Dent Res 89(10): 1063-1067.

3. Carvalho RM, Manso AP, Geraldeli S, Tay FR, Pashley DH (2012) Durability of bonds and clinical success of adhesive restorations. Dent Mater 28(1): 72-86.

4. Cardoso MV, Neves A, Mine A, Coutinho E, Landuyt K, et al. (2011) Current aspects on bonding effectiveness and stability in adhesive dentistry. Aust Dent J 56(Suppl 1): 31-44.

5. Breschi L, Mazzoni A, Ruggeri A, Cadenaro M, Lenarda R, et al. (2008) Dental adhesion review: Aging and stability of the bonded interface. Dent Mater 24(1): 90-101.

6. Fung DT, Wang VM, Laudier DM, Shine JH, Pljakic JB, et al. (2009) Sub rupture tendon fatigue damage. J Ortho Res 27: 264-273.

7. Betancourt DE, Baldion PA, Castellanos JE (2019) Resin-dentin bonding interface: mechanisms of degradation and strategies for stabilization of the hybrid layer. Int J Biomater pp. 1-11.

8. Perdigão J, Reis A, Loguercio AD (2013) Dentin adhesion and MMPs: A comprehensive review. J Esthet Restor Dent 25(4): 219-241. 
9. Tjäderhane L, Larjava H, Sorsa T, Uitto VJ, Larmas M, et al. (1998) The activation and function of host matrix metalloproteinases in dentin matrix breakdown in caries lesions. J Dent Res 77(8): 1622-1629.

10. Loguercio AD, Moura SK, Pellizzaro A, Bianco KD, Patzlaff RT, et al. (2008) Durability of enamel bonding using two-step self-etch systems on ground and unground enamel. Oper Dent 33(1): 79-88.

11. Tanaka J, Ishikawa K, Yatani H, Yamashita A, Suzuki K (1999) Correlation of dentin bond durability with water absorption of bonding layer. Dent Mater 18(1): 11-18.

12. Cai J, Palamara JEA, Burrow MF (2018) Effects of collagen crosslinkers on dentine: A literature review. Calcif Tissue Int 102(3): 265-279.

13. Feitosa VP, Pomacondor HC, Ogliari FA, Leal F, Correr AB, et al. (2014) Chemical interaction of 10-MDP (methacryloyloxy-decyldihydrogenphosphate) in zinc-doped self-etch adhesives. J Dent 42(3): 359-365.

14. Lee J, Sabatini C (2017) Glutaraldehyde collagen cross-linking stabilizes resin- dentin interfaces and reduces bond degradation. Eur J Oral Sci 125(1): 63-71.

15. Han B, Jaurequi J, Tang BW, Nimni ME (2003) Proanthocyanidin: a natural crosslinking reagent for stabilizing collagen matrices. J Biomed Mater Res A 65(1): 118-124.

16. Balalaie A, Rezvani MB, Basir MM (2018) Dual function of proanthocyanidins as both MMP inhibitor and crosslinker in dentin biomodification: A literature review. Dent Mater J 37(2): 173-182.

17. Castellan CS, Pereira PN, Grande RH, Bedran AK (2010) Mechnical characterization of proanthocyanidin dentin matrix interaction. Dent Mater 26(10): 968-973.

18. Epasinghe DJ, Yiu CK, Burrow MF, Tay FR, King NM (2012) Effect of proanthocyanidin incorporation into dental adhesive resin on resindentin bond strength. J Dent Res 40(3): 173-180.

19. Tezvergil MA, Agee KA, Hoshika T, Uchiyama T, Tjaderhane L, et al. (2011) Inhibition of MMPs by alcohols. Dent Mater 27(9): 926-933.

20. Kim DS, Kim J, Choi KK, Kim SY (2011) The influence of chlorhexidine on the remineralization of demineralized dentin. J Dent 39(12): 855-862.

21. Stanislawczuk R, Pereira F, Munoz MA, Luque I, Farago PV, et al. (2014) Effects of chlorhexidine-containing adhesives on the durability of resindentine interfaces. J Dent 42(1): 39-47.

22. Osorio R, Erhardt MC, Pimenta LAF, Toledano M (2005) EDTA treatment improves resin-dentin bond's resistance to degradation. J Dent Res 84(8): 736-740.

23. Breschi L, Martin P, Mazzoni A, Nato F, Carillho M (2010) Use of a specific MMP- inhibitor (galardin)for preservation of hybrid layer. Dent Mater 26(6): 571-578.

24. Acharya MR, Venitz J, Figg WD, Sparreboom A (2004) Chemically modified tetracyclines as inhibitors of matrix metalloproteinases. Drug Resist Updat 7(3): 195-208.

25. Bedran AK, Vidal CM, Dos PH, Castellan CS (2010) Long-term effect of carbodiimide on dentin matrix and resin-dentin bonds. J Biomed Mater Res B Appl Biomater 94(1): 250-255.

26. Tezvergil MA, Mutluay MM, Agee KA, Seseogullari DR, Hoshika T, et al. (2012) Carbodiimide cross-linking inactivates soluble and matrix-bound MMPs, in vitro. J Dent Res 91(2): 192-196.

27. Nikhil V, Jaiswal S, Bansal P, Arora R, Raj S, et al. (2016) Effect of phytic acid, ethylenediaminetetraacetic acid, and chitosan solutions on microhardness of the human radicular dentin. J Cons Dent 19(2): 179183.

28. Islam S, Hiraishi N, Nassar M, Yiu C, Otsuki M, et al. (2012) Effect of natural cross- linkers incorporation in a self- etching primer on dentin bond strength. J Dent 40(12): 1052-1059.

29. Bedran AK, Pereira PN, Duarte WR, Drummond JL, Yamauchi M (2007) Application of cross-linkers to dentin collagen enhances the ultimate tensile strength. J Biomed Mater Res B Appl Biomater 80(1): 268-272.

30. Sabatini C, Ortiz PA, Pashley DH (2015) Preservation of resin-dentin interfaces treated with benzalkonium chloride adhesive blends. Eur J Oral Sci 123(2): 108-115.

31. Tezvergil Mutluay A, Mutluay MM, Gu LS, Zhang K, Agee KA, et al. (2011) The anti-MMP activity of benzalkonium chloride. J Dent 39(1): 57-64.

32. Cova A, Breschi L, Nato F, Ruggeri A, Carrilho M, et al. (2011) Effect of UVA-activated riboflavin on dentin bonding. J Dent Res 90(12): 14391445.

33. Sasaki RT, Florio FM, Basting RT (2009) Effect of 10\% sodium ascorbate and $10 \%$ atocopherol in different formulations on the shear bond strength of enamel and dentin submitted to a home-use bleaching treatment. Oper Dent 34(6): 746-752.

34. Soeno K, Taira Y, Jimbo R, Sawase T (2008) Surface treatment with ascorbic acid and ferric chloride improves the micro-tensile bond strength of 4-META/MMATBB resin to dentin. J Dent 36(11): 940-944.

35. Vongphan N, Senawongse P, Somsiri W, Harnirattisai C (2005) Effects of sodium ascorbate on microtensile bond strength of total-etching adhesive system to $\mathrm{NaOCl}$ treated dentine. J dent 33(8): 689-695.

36. Mutluay TA, Agee KA, Mazzoni A, Carvalho RM, Tersariol IL, et al. (2015) Can quaternary ammonium methacrylates inhibit matrix MMPs and cathepsins. Dent Mater 31(2): e25-32.

37. Chauhan K, Basavanna RS, Shivanna V (2015) Effect of bromelain enzyme for dentin deproteinization on bond strength of adhesive system. J Cons Dent 18(5): 360-363.

38. Maeda YM, Ema K, Monobe M, Tokuda Y (2012) Epicatechin-3-0-(3\|-0 methyl)- gallate content in various tea cultivars and its in vitro inhibitory effect on histamine release. J Agric Food Chem 60(9): 2165-2170.

39. Mira L, Fernandez MT, Santos M, Rocha R, Jennings KR, et al. (2002) Interactions of flavonoids with iron and copper ions: a mechanism for their antioxidant activity. Free Radic Res 36(11): 1199-1208.

40. McCue PP, Shetty K (2004) Inhibitory effect of rosmarinic acid extracts on porcine pancreatic amylase in vitro. Asia Pacific J Clin Nur 13(1): 101106.

41. Omanakuttan A, Nambiar J, Harris RM, Bose C, Varghese RK (2012) Anacardic acid inhibits the catalytic activity of matrix metalloproteinase -2 and matrix metalloproteinase-9. Mol Pharmacol 82(4): 614-622.

42. Moreira MA, Souza NO, Sousa RS, Freitas DQ Lemos MV, et al. (2017) Efficacy of new natural biomodification agents from Anacardiaceae extracts on dentin collagen cross-linking. Dent Mater 33(10): 11031109.

43. Epasinghe DJ, Yiu CK, Burrow MF, Tsoi JKH, Tay FR (2014) Effect of flavanoids on the mechanical properties of demineralised dentin. J Dent 42(9): 1178-1184.

44. Li J, Chen B, Hong N, Wu S, Li Y (2018) Effect of baicalein on matrix metalloproteinases and durability of resin-dentin bonding. Oper Dent 43(4): 426-436. 